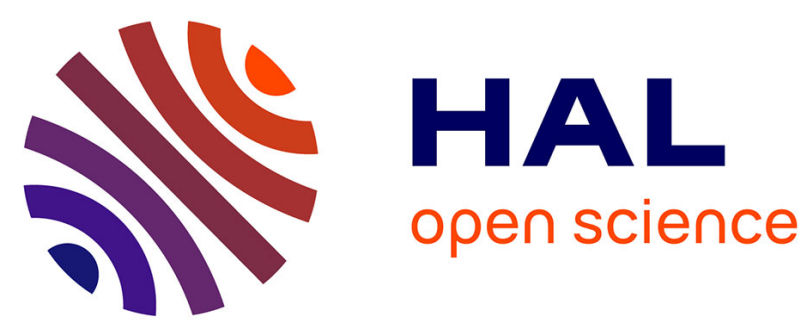

\title{
Hyperspectral Estimation of Nitrogen Content in Winter Wheat Leaves Based on Unmanned Aerial Vehicles
} Liu Mingxing, Li Changchun, Feng Haikuan, Pei Haojie, Li Zhenhai, Yang Fuqin, Yang Guijun, Xu Shouzhi

\section{- To cite this version:}

Liu Mingxing, Li Changchun, Feng Haikuan, Pei Haojie, Li Zhenhai, et al.. Hyperspectral Estimation of Nitrogen Content in Winter Wheat Leaves Based on Unmanned Aerial Vehicles. 11th International Conference on Computer and Computing Technologies in Agriculture (CCTA), Aug 2017, Jilin, China. pp.321-339, 10.1007/978-3-030-06179-1_33 . hal-02111529

\author{
HAL Id: hal-02111529 \\ https://hal.inria.fr/hal-02111529
}

Submitted on 26 Apr 2019

HAL is a multi-disciplinary open access archive for the deposit and dissemination of scientific research documents, whether they are published or not. The documents may come from teaching and research institutions in France or abroad, or from public or private research centers.
L'archive ouverte pluridisciplinaire HAL, est destinée au dépôt et à la diffusion de documents scientifiques de niveau recherche, publiés ou non, émanant des établissements d'enseignement et de recherche français ou étrangers, des laboratoires publics ou privés.

\section{(c)(1)}

Distributed under a Creative Commons Attribution| 4.0 International License 


\title{
Hyperspectral Estimation of Nitrogen Content in Winter Wheat Leaves Based on Unmanned Aerial Vehicles
}

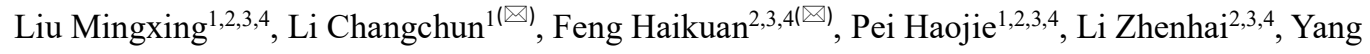 \\ Fuqin $^{5}$,Yang Guijun ${ }^{2,3,4}, \mathrm{Xu}$ Shouzhi ${ }^{6}$ \\ ${ }^{1}$ School of Surveying and Land Information Engineering, Henan Polytechnic University, Jiaozuo 454000, \\ China \\ \{liumingxingreal, lichangchun610\}@126.com, \\ xmljphj@163.com \\ ${ }^{2}$ National Engineering Research Center for Information Technology in Agriculture, Beijing,100097, China \\ \{fenghaikuan123, yangfuqin0202, guijun.yang\}@163.com, \\ lizh323@126. com
}

${ }^{3}$ Key Laboratory for Information Technologies in Agriculture, the Ministry of Agriculture, 100097, China

${ }^{4}$ Beijing Engineering Research Center of Agricultural Internet of Things, Beijing, 100097, China

${ }^{5}$ College of Civil Engineering, Henan Institute of Engineering, Zhengzhou 451191, China

${ }^{6}$ National Calibration Center for Surveying Instruments,Beijing,100039, China

xsz2011@whu.edu.cn

\begin{abstract}
Leaf nitrogen content is an important index of crop growth and plays an important role in crop growth and development. In this paper, the hyperspectral data of winter wheat and the leaf nitrogen content is used to study winter wheat on flagging stage, flowering stage and grain filling stage. The estimation model of nitrogen content in winter wheat leaves at different growth stages is constructed by using partial least squares method and verified by using a cross-validation method. The results showed that R2 and the RMSE of the three growth stages were 0.53, 0.68, 0.64 and $0.331 \%, 0.246 \%$ and $0.406 \%$ respectively, and R2 and RMSE of model validation were $0.44,0.71$, 0.64 and $0.369 \%, 0.235 \%$ and $0.410 \%$. Both the prediction model and the verification model had high reliability. Therefore, it is feasible for UAV to carry hyperspectral monitoring system for retrieving nitrogen content of winter wheat leaves.
\end{abstract}

Keywords: unmanned aerial vehicles, hyperspectral, winter wheat, leaf nitrogen content, partial least squares method

\section{Introduction}

Nitrogen is the most important mineral nutrient element in crop life, which can promote the growth of vegetative organs such as roots, stems and leaves, expand the area of photosynthesis and enhance the accumulation of photosynthetic products [1-2]. The nitrogen content directly affects crop growth and 
yield [3].Inversion of crop nitrogen content by remote sensing system is one of the quantitative agricultural remote sensing hotspots. In order to establish the relationship between wheat nitrogen content and spectrum, domestic and foreign scholars have studied the changes of sensitive spectral bands in different growth stages of wheat [4-5]. Zhang Xiaoyuan et al.[6] put forward the sensitivity study of retrieving leaf nitrogen content of Winter Wheat with different spectral vegetation index. The results showed that Using Hyperspectral Vegetation index can effectively achieve nitrogen content inversion of crop leaves. Zhu Xinkai et al. [7] have put forward a preliminary study on the use of SPAD to predict chlorophyll and nitrogen content in wheat leaves. It turned out that the SPAD value of wheat leaves is positively correlated with total nitrogen content, and the total nitrogen content could be estimated by SPAD value for wheat nitrogen nutrition. Li Yingxue et al. [8] have proposed a quantitative relationship between nitrogen content and canopy reflectance index in wheat leaves,and the results showed that the red edge position of canopy reflectance spectrum could indicate the leaf nitrogen content of wheat varieties with different protein types. Sun Yanxin et al. [9] have raised the establishment and validation of GRNN hyperspectral remote sensing model for retrieving nitrogen content in winter wheat leaves based on GA. The results showed that the selected spectral parameters and the corresponding model could reflect the relationship between total nitrogen in wheat leaves, and the effect is better than the stepwise regression model. Zhang Guosheng et al. [10] proposed rice leaf nitrogen Hyperspectral Estimation and inversion based on the model. Results showed that there is a good correlation between leaf nitrogen content and tillering stage normalized vegetation index. Wang Jihua et al. [11] have put forward to use wheat canopy reflectance spectra to retrieve the vertical distribution of nitrogen by PLS algorithm, and the results showed that it is feasible to use PLS algorithm to estimate the vertical distribution of total nitrogen in wheat leaves. Ju Changhua [12] has used land-air hyperspectral remote sensing to monitor the nitrogen status and growth characteristics of wheat. The results showed that the sensitive bands of leaf nitrogen are mainly in the visible and nearinfrared bands, among which the red-edge region is the most significant. Zhai Qingyun et al. [13] have raised hyperspectral differences and monitoring model construction of nitrogen content in wheat leaves based on different soil texture, and it turned out that using NDSI (FD710, FD690), DSI (R515, R460) and RSI (R535, R715) as independent variables, the estimation model can well predict the nitrogen content of wheat leaves in sandy, loamy and clay 3 germplasms. Li Fenling et al. [14] have proposed the estimation of nitrogen content in winter wheat leaves with wide band reflectance of the simulated multispectral satellite. The results showed that the spectral index based on the composite index (TCARI/OSAVI) and the transformed chlorophyll absorption index (TCARI) has some advantages in monitoring the nitrogen content of wheat leaves during the whole growth period. Wang Renhong et al. [15] proposed to estimate the nitrogen nutrient index of Winter Wheat Based on hyperspectral. The results showed that the linear interpolation of the red edge position, the red edge correction single index, ratio index, simple ratio pigment index, index and spectral parameters in nitrogen have a good correlation, can be used for inversion of canopy nitrogen nutrition level. Yang Fuqin et al. [16] have put forward the optimal regression model of plant nitrogen content at different growth stages based on the importance of variable projection - partial least squares - Akai information criterion integration model, and the results showed that the flag stage is the best period for monitoring the nitrogen nutrition of Winter Wheat by hyperspectral remote sensing. H Liu et al. [17] have raised quantitative modeling for leaf nitrogen content of winter wheat using UAV-based hyperspectral data. The results showed that the predicted values are very good in jointing stage, flagging leaf stage, and flowering stage, while it is a little bit less in the filling stage. 
This essay first analyzed the correlation between the original spectrum, the first derivative of the spectrum, the normalized spectral index (NDSI), the ratio spectral index(RSI) and the leaf nitrogen content, and then each growth period selects the spectra of the original spectral positive correlation maximum and negative correlation maximum ,the spectra of first derivative positive correlation maximum and negative correlation maximum , a group of spectra with the greatest NDSI correlation value and a group of spectra with the greatest RSI correlation value, with a total of six parameters. Next, the estimation model of nitrogen content in winter wheat leaves is constructed by partial least squares method and verified by cross-validation method. So as to provide a new technical approach for hyperspectral remote sensing inversion of nitrogen content in winter wheat leaves.

\section{Materials and methods}

\subsection{Brief Introduction of Research Area}

Field experiments were conducted at the National Precision Agriculture Research and demonstration base from 2014 to 2015 . The base is located in the northeast of Beijing City,located at latitude $40^{\circ} 00^{\prime}-$ $40^{\circ} 21^{\prime}$, longitude $116^{\circ} 34^{\prime}-117^{\circ} 00^{\prime}$. The Base covers an area of about 2500 acres, altitude $36 \mathrm{~m}$, fertile land, flat terrain, and soil type is fluvo-aquic soil. The climate in the base area belongs to warm temperate semi-humid continental monsoon weather, four distinct seasons, cold and dry in winter, hot and rainy summer.The average temperature is $13^{\circ} \mathrm{C}$, and the average annual precipitation is $510 \mathrm{~mm}$. At the end of September, early winter wheat is planted here.

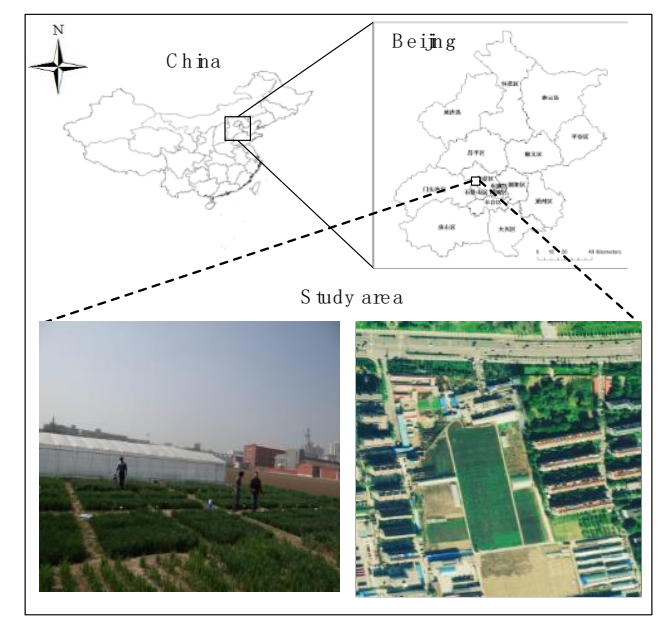

Fig.1 National Precision Agriculture Research and Demonstration Base

\subsection{Experimental design}

Using varieties, water, nitrogen fertilization orthogonal test; Wheat is divided into two varieties:

Beijing 9843 (J9843), Zhongmai 175 (ZM175); There were 4 levels of fertilizer: 0 kg urea / acre (N1), $13 \mathrm{~kg}$ urea / acre (N2), $26 \mathrm{~kg}$ urea / acre (N3), $39 \mathrm{~kg}$ urea /acre (N4); Planting plots were processed 16 times and repeated 3 times. Field planting map (see below): From east to west, the total length is $84 \mathrm{~m}$, and the total length is $32 \mathrm{~m}$ from south to North; The number of planting area is 48 , and each area is $6 \mathrm{~m} * 8 \mathrm{~m}$. 


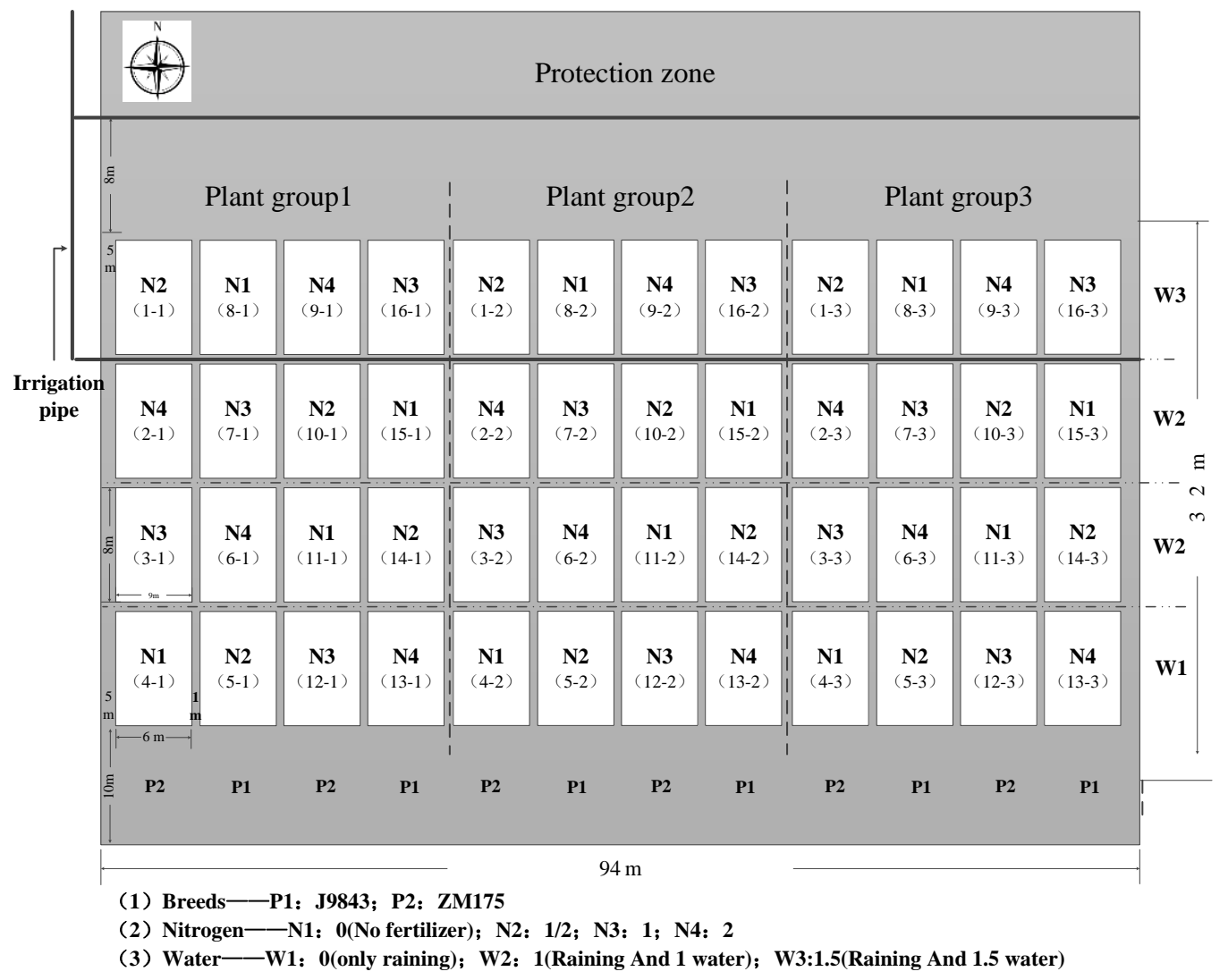

Fig.2 Test plan

\subsection{Data acquisition and processing}

\subsubsection{Leaf nitrogen content data acquisition}

The nitrogen content of winter wheat leaves is collected at flagging stage (April 27, 2015), flowering stage (May 11, 2015) and grain filling stage (May 25, 2015). Twenty growing average winter wheat plants were selected as experimental samples for each plot. Firstly, The wheat samples were collected in paper bags and the leaves of wheat should be integral during collection. Next cut the wheat sample leaves separately in the test paper bag, and Put the paper bag in the $105 \mathrm{C}$ oven for half an hour. Then we reduced oven temperature immediately and maintained oven temperature of 80 degrees $\mathrm{C}$ for drying samples, drying for more than 24 hours, until the quality of the sample remained unchanged. After drying, the sample blades were weighed and crushed, and the nitrogen content of the sample blades is measured by Kjeldahl apparatus. The nitrogen content of the sample leaves is the product of the nitrogen mass fraction and the sample biomass. Finally, the average nitrogen content of winter wheat leaves in each plot is taken as the value of nitrogen content in winter wheat leaves.

\subsubsection{Hyperspectral data acquisition}

The experiment used UAV equipped with a UHD-185 remote sensor to sample spectral of winter wheat from 10am to 2pm Beijing time in Changping districts when the weather is sunny and windless. The hyperspectral remote sensing images were collected at flagging stage (April 27, 2015), flowering stage (May 11, 2015) and grain filling stage (May 25, 2015), using the same flight route. The UHD-185 hyperspectral measures a range of 454 to $950 \mathrm{~nm}$, a sampling interval of $4 \mathrm{~nm}$, and a cubic resolution of one megapixel. The spectral range of the spectrometer is narrow, and the spectral resolution of the visible light to near-infrared band is nanometer order, and the number of spectral bands is large, and the 
band is continuous, which can meet the requirement of inversion of nitrogen content in winter wheat leaves. The UHD-185 hyperspectral sensor carried by the unmanned aerial vehicle (UAV) had been denoised and lens distortion correction before used.

The experiment used Agisoft photoscan software to splice UAV hyperspectral remote image. The pre-processed hyperspectral images were introduced into the ArcGIS software, and the rectangular region of interest is selected from the hyperspectral remote sensing image in combination with the 48 experimental plots. Finally, the average spectral reflectance of each experimental plot is extracted to obtain 125 spectral average reflectances at wavelengths 454 to $950 \mathrm{~nm}$.

\subsection{Method}

\subsubsection{Vegetation Index Selection}

The normalized difference index NDSI [18] and the ratio spectral index RSI [19] were constructed by referring to the normalized vegetation index and the ratio vegetation index in the study of vegetation canopy spectra. These formulas are as follows:

$$
\begin{gathered}
\operatorname{NDSI}\left(\lambda_{1}, \lambda_{2}\right)=\frac{R_{\lambda 2}-R_{\lambda 1}}{R_{\lambda 2}+R_{\lambda 1}} \\
\operatorname{RSI}\left(\lambda_{1}, \lambda_{2}\right)=\frac{R_{\lambda 2}}{R_{\lambda 1}}
\end{gathered}
$$

Where $\lambda_{1}$ is wavelength 1 and $\lambda_{2}$ is wavelength 2. $R_{\lambda 1}$ represents spectral reflectance of winter wheat leaves at wavelength 1 , and $R_{\lambda 2}$ represents spectral reflectance of winter wheat leaves at wavelength 2 .

\subsubsection{Partial least squares method}

Partial least squares [20] is a multivariate statistical data analysis method that minimizes the square of the error and finds the best matching function model for a set of data. It is generally used to study the regression model of dependent variables for multiple independent variables. The simplest form is the linear relationship between the variable $\mathrm{Y}$ and the independent variable $\mathrm{X}$, the expression is:

$$
Y=a_{0}+a_{1} X_{1}+a_{2} X_{2}+\mathrm{L}+a_{n} X_{n}
$$

Where $\mathrm{a} 0$ is the intercept of the regression coefficient; ai is the regression coefficient; $\mathrm{Xi}$ is the independent variable $1 \sim \mathrm{n}$.

\subsection{Statistical Analysis}

In this study, determination coefficient (R2) and root-mean-square error were regarded as indicators to interpret and quantify the relationship between LNC and vegetation index. Generally speaking, the higher R2, the smaller RMSE, and the better ability of the estimated model between the LNC and vegetation index, otherwise, the estimated ability is poor. 


\section{Data processing and analysis}

\subsection{Analysis of correlation between original spectrum and leaf nitrogen content}

The correlation between the average reflectance of the arbitrary band spectrum and the nitrogen content of the corresponding wheat leaves is analyzed in the winter wheat flagging stage, flowering stage and grain filling stage, and the graphs of wavelength and correlation coefficient were obtained. Each growth period sorted the correlation coefficients and selected the spectra of the original spectral positive correlation maximum and negative correlation maximum.

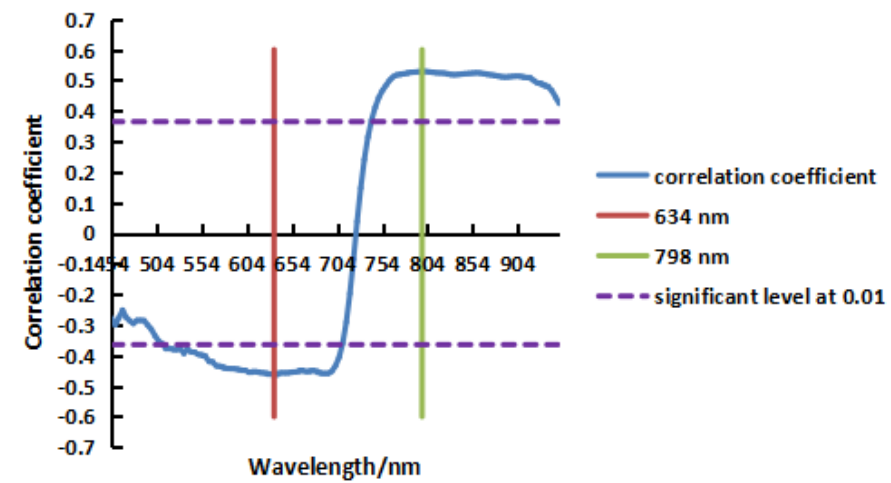

Fig.3 Correlation between original spectrum and leaf nitrogen content at flagging stage

As shown in Figure 3, In the range of 514 to $706 \mathrm{~nm}$, the leaf nitrogen content is significantly negatively correlated with the original spectrum, and the correlation coefficient in the $634 \mathrm{~nm}$ band is the best, and the correlation coefficient is -0.46 . In the range of 742 to $950 \mathrm{~nm}$, the leaf nitrogen content is significantly positively correlated with the original spectrum, and the correlation coefficient in the $798 \mathrm{~nm}$ band is the best, and the correlation coefficient is $0.53 .634 \mathrm{~nm}$ band and $798 \mathrm{~nm}$ band are selected as the parameters for modeling the flagging stage of winter wheat.

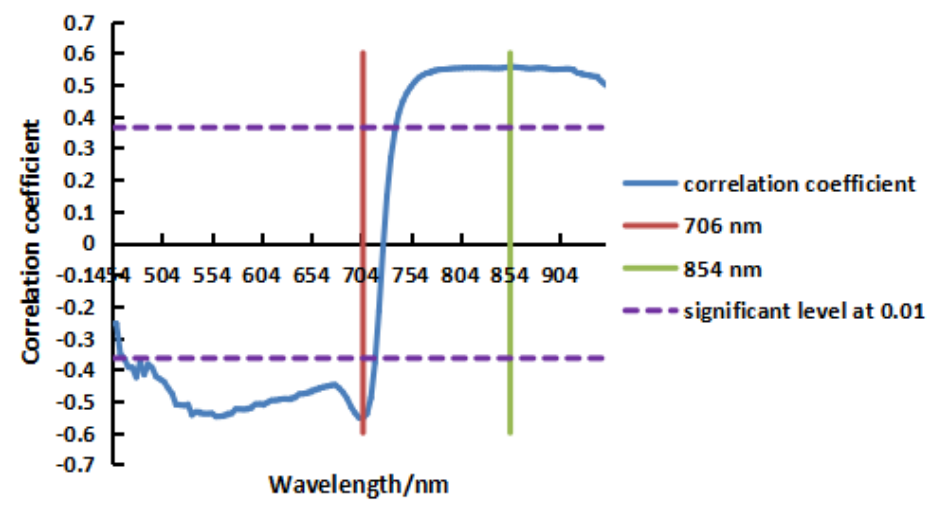

Fig.4 Correlation between original spectrum and leaf nitrogen content at flowering stage

As shown in Figure 4, In the range of 466 to $718 \mathrm{~nm}$, the leaf nitrogen content is significantly negatively correlated with the original spectrum, and the correlation coefficient in the 706nm band is the best, and the correlation coefficient is -0.55 . In the range of 742 to $950 \mathrm{~nm}$, the leaf nitrogen content is significantly positively correlated with the original spectrum, and the correlation coefficient in the 
$854 \mathrm{~nm}$ band is the best, and the correlation coefficient is $0.56 .706 \mathrm{~nm}$ band and $854 \mathrm{~nm}$ band are selected as the parameters for modeling the flowering stage of winter wheat.

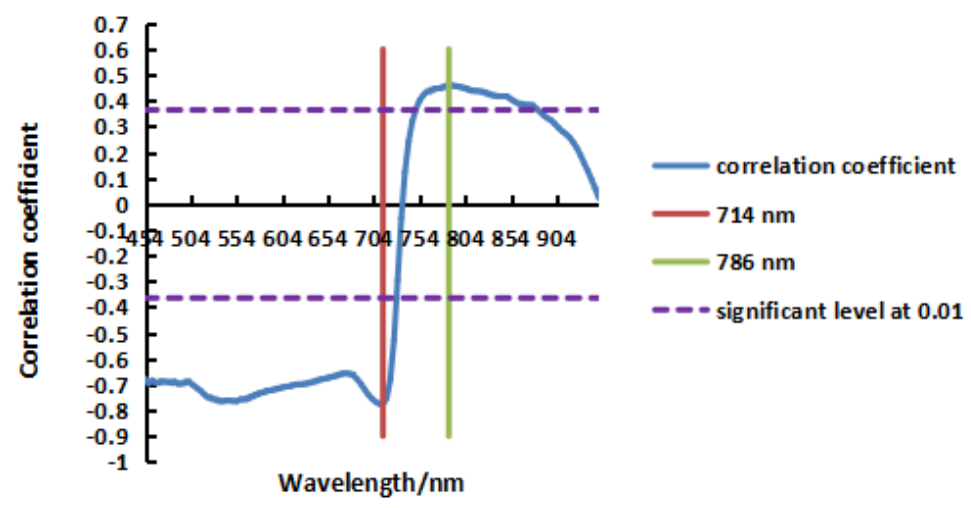

Fig.5 Correlation between original spectrum and leaf nitrogen content at grain filling stage

As shown in Figure 5, In the range of 454 to $726 \mathrm{~nm}$, the leaf nitrogen content is significantly negatively correlated with the original spectrum, and the correlation coefficient in the $714 \mathrm{~nm}$ band is the best, and the correlation coefficient is -0.78 . In the range of 750 to $882 \mathrm{~nm}$, the leaf nitrogen content is significantly positively correlated with the original spectrum, and the correlation coefficient in the $786 \mathrm{~nm}$ band is the best, and the correlation coefficient is $0.46 .714 \mathrm{~nm}$ band and $786 \mathrm{~nm}$ band are selected as the parameters for modeling the grain filling of winter wheat.

\subsection{Analysis of correlation between first derivative of spectrum and leaf nitrogen content}

The correlation between the first derivative of spectral average reflectance of the arbitrary band spectrum and the nitrogen content of the corresponding wheat leaves was analyzed in the winter wheat flagging stage, flowering stage and grain filling stage, and the graphs of wavelength and correlation coefficient were obtained. Each growth period Sorted the correlation coefficients and selected the spectra of first derivative positive correlation maximum and negative correlation maximum.

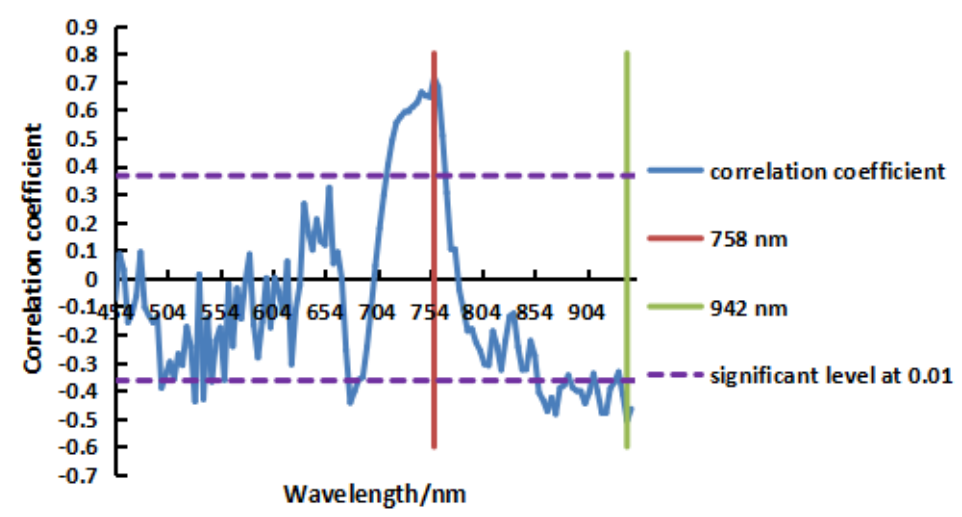

Fig.6 Correlation between first derivative of spectrum and nitrogen content in leaves at flagging stage

As shown in Figure 6, In the range of 714 to $766 \mathrm{~nm}$, the leaf nitrogen content is significantly positively correlated with the first derivative of the spectrum, and the correlation coefficient in the $758 \mathrm{~nm}$ band is the best, and the correlation coefficient is 0.71 . In the range of 858 to $882 \mathrm{~nm}, 890$ to 
906nm, 914 to $930 \mathrm{~nm}, 938$ to $930 \mathrm{~nm}$ and other sporadic spectrum, the leaf nitrogen content is significantly negatively correlated with the first derivative of the spectrum, and the correlation coefficient in the $942 \mathrm{~nm}$ band is the best, and the correlation coefficient is $-0.51 .758 \mathrm{~nm}$ band and $942 \mathrm{~nm}$ band are selected as the parameters for modeling the flagging stage of winter wheat.

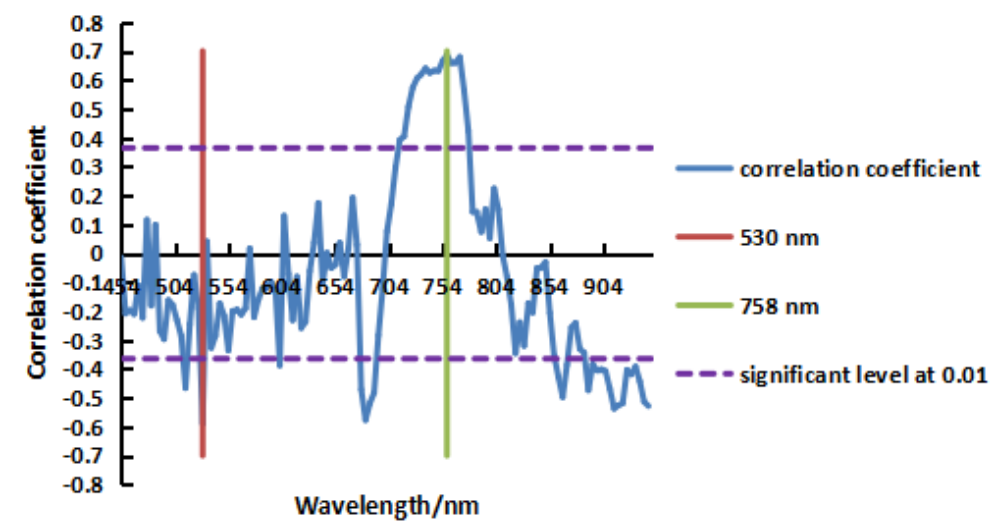

Fig.7 Correlation between first derivative of spectrum and nitrogen content in leaves at flowering stage

As shown in Figure 6, In the range of 714 to $778 \mathrm{~nm}$, the leaf nitrogen content is significantly positively correlated with the first derivative of the spectrum, and the correlation coefficient in the $758 \mathrm{~nm}$ band is the best, and the correlation coefficient is 0.69 . In the range of $530 \mathrm{~nm}, 678$ to $690 \mathrm{~nm}$, 862 to $870 \mathrm{~nm}, 890$ to $946 \mathrm{~nm}$ and other sporadic spectrum, the leaf nitrogen content is significantly negatively correlated with the first derivative of the spectrum, and the correlation coefficient in the $530 \mathrm{~nm}$ band is the best, and the correlation coefficient is $-0.59 .530 \mathrm{~nm}$ band and $758 \mathrm{~nm}$ band are selected as the parameters for modeling the flowering stage of winter wheat.

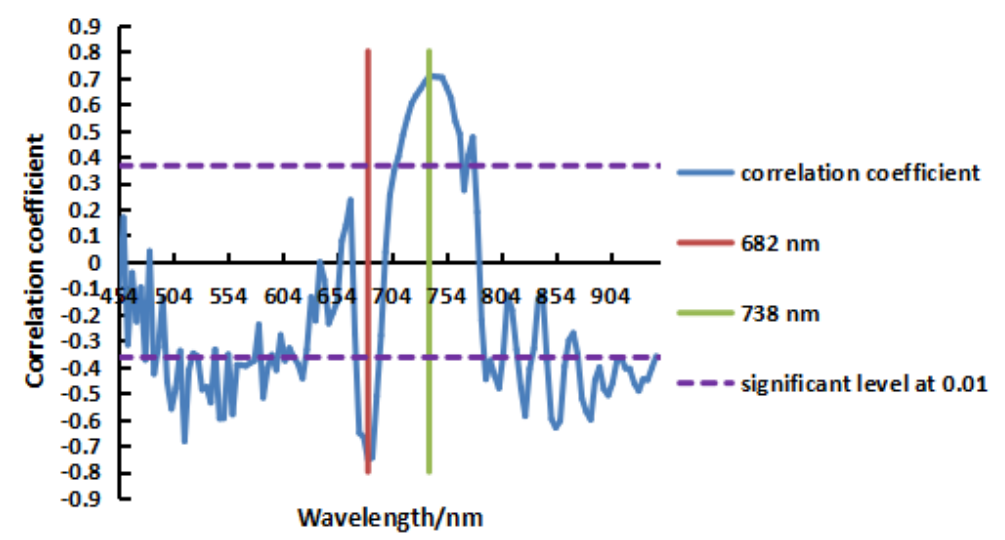

Fig.8 Correlation between first derivative of spectrum and nitrogen content in leaves at grain

$$
\text { filling stage }
$$

As shown in Figure 7, In the range of 710 to $766 \mathrm{~nm}$ and 744 to $778 \mathrm{~nm}$, the leaf nitrogen content is significantly positively correlated with the first derivative of the spectrum, and the correlation coefficient in the $738 \mathrm{~nm}$ band is the best, and the correlation coefficient is 0.71 . In the range of 558 to $578 \mathrm{~nm}, 674$ to $690 \mathrm{~nm}, 790$ to $802 \mathrm{~nm}, 846$ to $862 \mathrm{~nm}, 878$ to $942 \mathrm{~nm}$ and other sporadic spectrum, the leaf nitrogen content is significantly negatively correlated with the first derivative of the spectrum, and the correlation coefficient in the $682 \mathrm{~nm}$ band is the best, and the correlation coefficient is $-0.75 .682 \mathrm{~nm}$ 
band and $730 \mathrm{~nm}$ band are selected as the parameters for modeling the grain filling stage of winter wheat.

\subsection{Analysis of correlation between NDSI and leaf nitrogen content}

Using the MATLAB program, the NDSI of the spectral reflectance of any two bands in the winter wheat flagging stage, flowering stage and grain filling stage was calculated. Then the coefficient of determination between NDSI and leaf nitrogen content is calculated, and the contour map of each growth period was obtained,as shown in Figures 9 through 11.

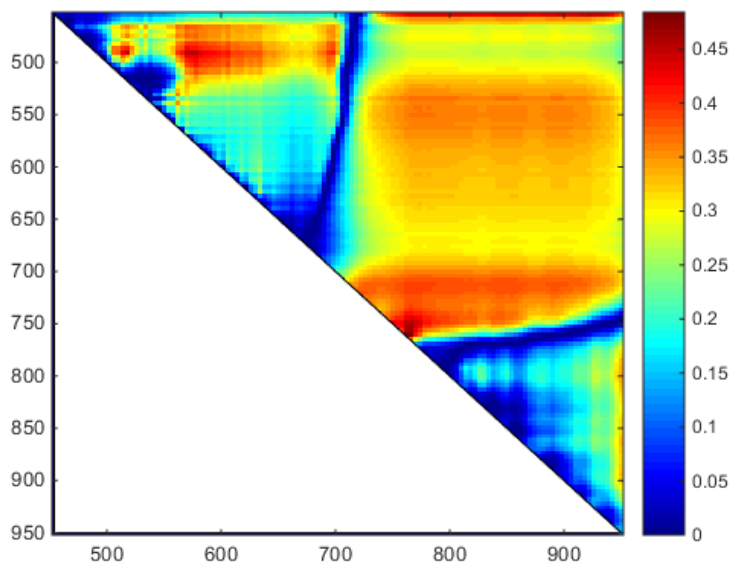

Fig.9 Coefficient of determination between NDSI and leaf nitrogen content at flagging stage

As shown in Figure 9, The leaf nitrogen content of winter wheat has a good correlation with NDSI in many bands during flagging stage. These bands are as follows: the range on the $\mathrm{x}$-axis is 746 to $758 \mathrm{~nm}$ and the range on the y-axis is 762 to $766 \mathrm{~nm}$. These regions show that the nitrogen content of leaf can be well estimated by using NDSI, and the coefficient of determination is above 0.45 . NDSI $[758,762]$ has the best correlation, with a coefficient of determination of 0.48 . NDSI[758,762] is selected as the parameter for modeling at the flagging stage of winter wheat.

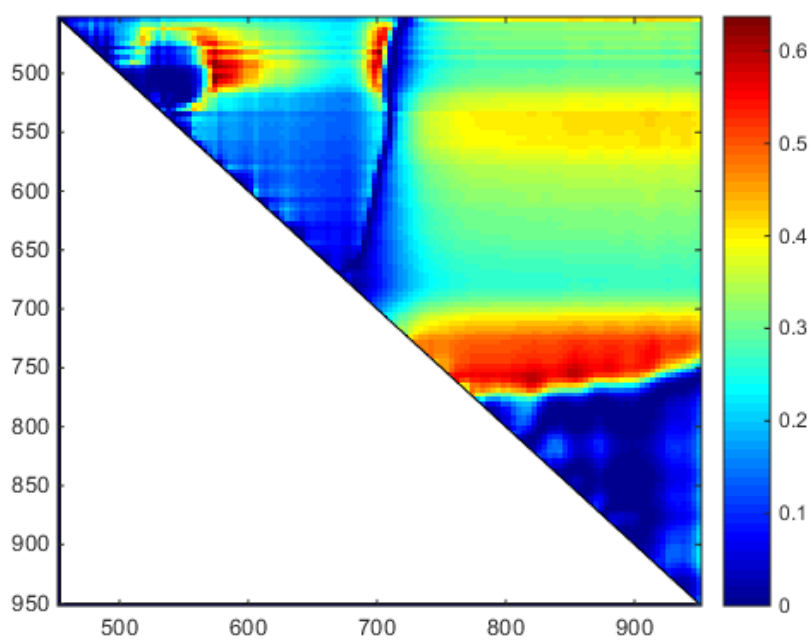

Fig.10 Coefficient of determination between NDSI and leaf nitrogen content at flowering stage 
As shown in Figure 10, The leaf nitrogen content of winter wheat has a good correlation with NDSI in many bands during flowering stage. These bands are as follows: the range on the $\mathrm{x}$-axis is 490 to $506 \mathrm{~nm}$ and the range on the y-axis is 574 to $578 \mathrm{~nm}$. These regions show that the nitrogen content of leaf can be well estimated by using NDSI, and the coefficient of determination is above 0.6 .

NDSI[502,574] has the best correlation, with a coefficient of determination of 0.64. NDSI[502,574] is selected as the parameter for modeling at the flowering stage of winter wheat.

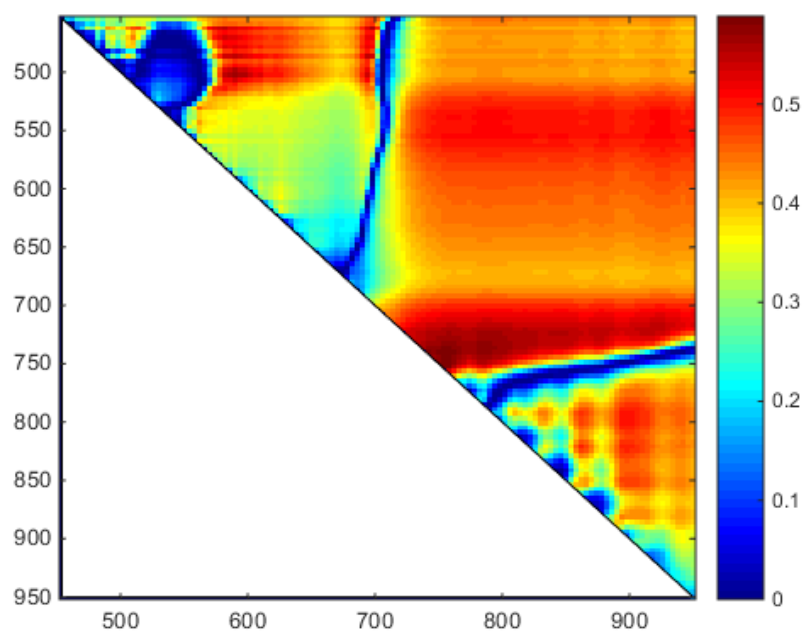

Fig.11 Coefficient of determination between NDSI and leaf nitrogen content at grain filling stage

As shown in Figure 11, The leaf nitrogen content of winter wheat has a good correlation with NDSI in many bands during grain filling stage. The band range is as follows: The range on the $\mathrm{x}$-axis is $502 \mathrm{~nm}$, and the range on the $\mathrm{y}$-axis is 586 to $598 \mathrm{~nm}$; the range on the $\mathrm{x}$-axis is 718 to $726 \mathrm{~nm}$, and the range on the $y$-axis is 750 to $850 \mathrm{~nm}$; The range on the $\mathrm{x}$-axis is 730 to $738 \mathrm{~nm}$, and the range on the $\mathrm{y}$ axis is 738 to $806 \mathrm{~nm}$; the range on the $\mathrm{x}$-axis is 742 to $750 \mathrm{~nm}$, and the range on the $y$-axis is 754 to $766 \mathrm{~nm}$. These regions show that the nitrogen content of leaf can be well estimated by using NDSI, and the coefficient of determination is above 0.55 . NDSI[746,754] has the best correlation, with a coefficient of determination of 0.59 . NDSI[746,754] is selected as the parameter for modeling at the flowering stage of winter wheat.

\subsection{Analysis of correlation between RSI and leaf nitrogen content}

Using the MATLAB program, the RSI of the spectral reflectance of any two bands in the winter wheat flagging stage, flowering stage and grain filling stage was calculated. Then the coefficient of determination between RSI and leaf nitrogen content is calculated, and the contour map of each growth period was obtained,as shown in Figures 12 through 14. 


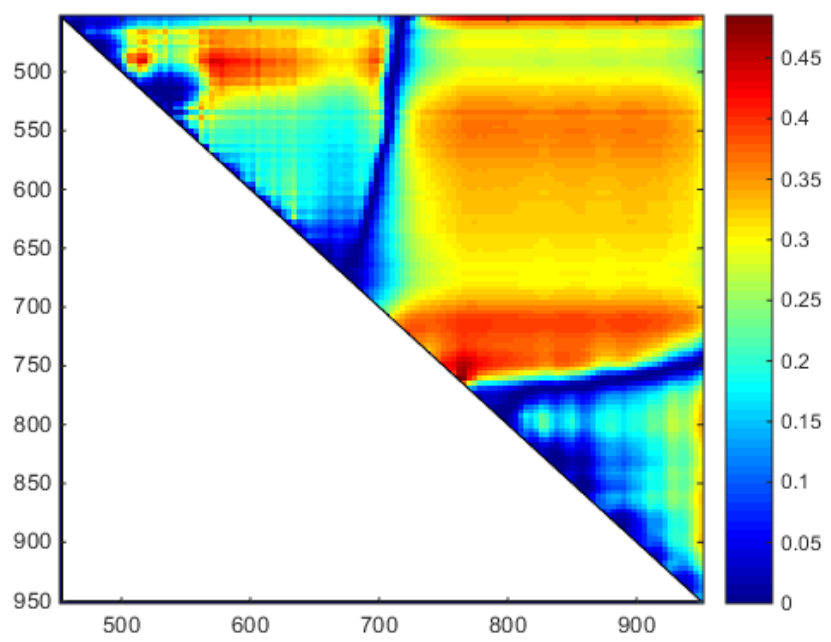

Fig.12 Coefficient of determination between RSI and leaf nitrogen content at flagging stage

As shown in Figure12, The leaf nitrogen content of winter wheat has a good correlation with RSI in many bands during flagging stage. These bands are as follows: the range on the $\mathrm{x}$-axis is 746 to $758 \mathrm{~nm}$ and the range on the y-axis is 762 to $766 \mathrm{~nm}$. These regions show that the nitrogen content of leaf can be well estimated by using RSI, and the coefficient of determination is above 0.45 . $\operatorname{RSI}[758,762]$ has the best correlation, with a coefficient of determination of 0.49 . RSI[758,762] is selected as the parameter for modeling at the flagging stage of winter wheat.

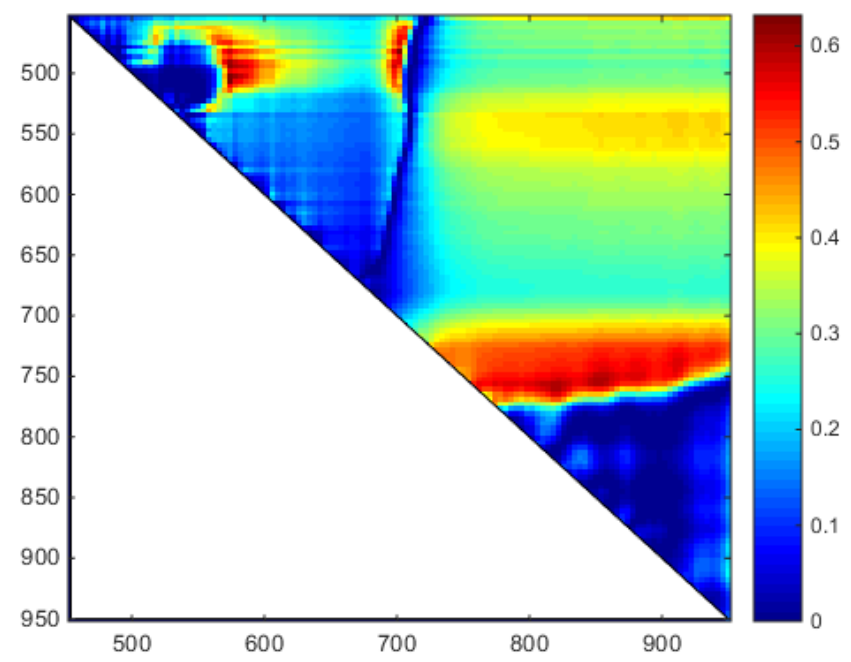

Fig.13 Coefficient of determination between RSI and leaf nitrogen content at flowering stage

As shown in Figure 13, The leaf nitrogen content of winter wheat has a good correlation with RSI in many bands during flowering stage. These bands are as follows: The range on the $\mathrm{x}$-axis is $482 \mathrm{~nm}$, and the range on the $y$-axis is $702 \mathrm{~nm}$; the range on the $x$-axis is $490 \mathrm{~nm}$, and the range on the $y$ axis is $574 \mathrm{~nm}$; The range on the $\mathrm{x}$-axis is $502 \mathrm{~nm}$, and the range on the y-axis is 574 to $578 \mathrm{~nm}$; the range on the $x$-axis is $508 \mathrm{~nm}$, and the range on the $y$-axis is $574 \mathrm{~nm}$. the range on the $\mathrm{x}$-axis is $570 \mathrm{~nm}$, and the range on the y-axis is $854 \mathrm{~nm}$. These regions show that the nitrogen content of leaf can be well estimated by using RSI, and the coefficient of determination is above 0.6 . RSI[502,574] has the best 
correlation, with a coefficient of determination of 0.63 . RSI[502,574] is selected as the parameter for modeling at the flowering stage of winter wheat.

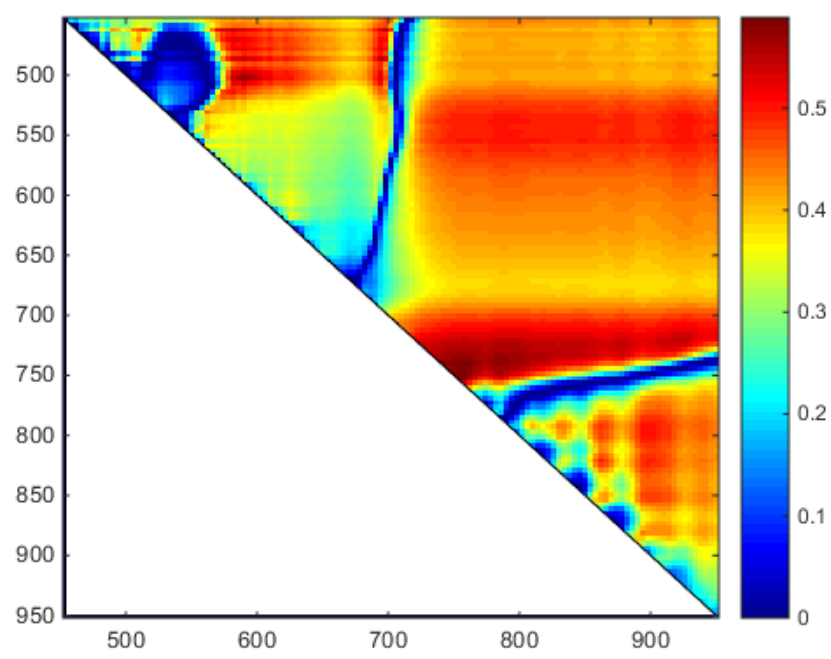

Fig.14 Coefficient of determination between RSI and leaf nitrogen content at grain filling stage

As shown in Figure 14, The leaf nitrogen content of winter wheat has a good correlation with RSI in many bands during flowering stage. These bands are as follows: The range on the $\mathrm{x}$-axis is $502 \mathrm{~nm}$, and the range on the $\mathrm{y}$-axis is 586 to $594 \mathrm{~nm}$; the range on the $\mathrm{x}$-axis is $722 \mathrm{~nm}$, and the range on the $y$-axis is 754 to $762 \mathrm{~nm}$; The range on the $x$-axis is $722 \mathrm{~nm}$, and the range on the $y$-axis is 782 to $802 \mathrm{~nm}$; the range on the $\mathrm{x}$-axis is 726 to $738 \mathrm{~nm}$, and the range on the $y$-axis is 742 to $806 \mathrm{~nm}$; the range on the $\mathrm{x}$-axis is 726 to $734 \mathrm{~nm}$, and the range on the $\mathrm{y}$-axis is 810 to $818 \mathrm{~nm}$. The range on the $\mathrm{x}$-axis is $742 \mathrm{~nm}$, and the range on the $y$-axis is 746 to $770 \mathrm{~nm}$; the range on the $\mathrm{x}$-axis is $742 \mathrm{~nm}$, and the range on the $y$-axis is 778 to $798 \mathrm{~nm}$; The range on the $\mathrm{x}$-axis is $746 \mathrm{~nm}$, and the range on the y-axis is 754 to $790 \mathrm{~nm}$. These regions show that the nitrogen content of leaf can be well estimated by using RSI, and the coefficient of determination is above 0.55 . RSI[502,574] has the best correlation, with a coefficient of determination of 0.59 . RSI[746,754] is selected as the parameter for modeling at the grain filling stage of winter wheat.

\section{Model building and accuracy analysis}

\subsection{Model building}

At each stage of growth, we first selected the spectra of the original spectral positive correlation maximum and negative correlation maximum ,the spectra of first derivative positive correlation maximum and negative correlation maximum, a group of spectra with the greatest NDSI correlation value and a group of spectra with the greatest RSI correlation value, with a total of six parameters. And then the partial least squares method was used to establish the estimation model of nitrogen content in leaves of winter wheat leaves at different growth stages. Finally, the relationship between the measured value and the predicted value was analyzed,as shown in Figures 15 through 17. 


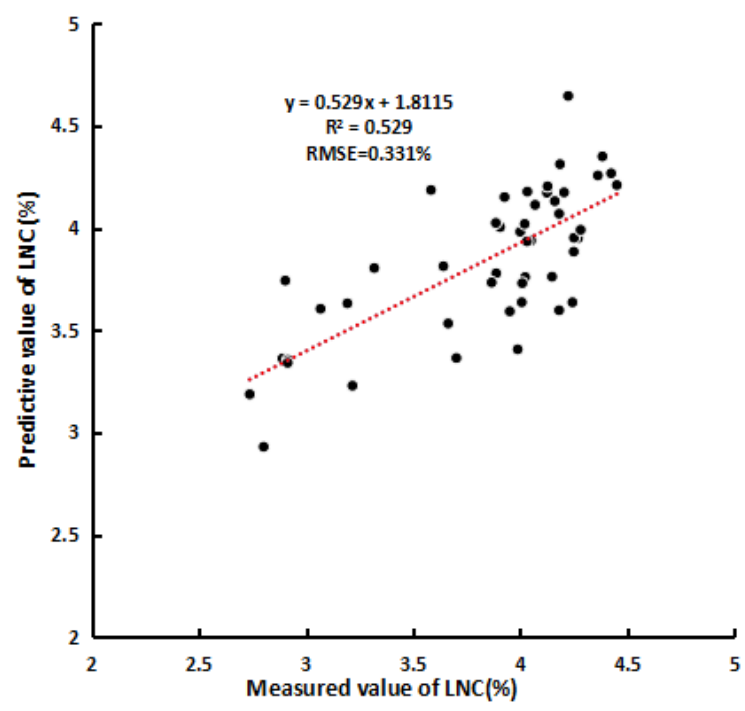

Fig.15 Relationship between predicted LNC and measured LNC at flagging stage

After correlation analysis between spectral and leaf nitrogen content, the original spectral trough $634 \mathrm{~nm}$ and the peak $798 \mathrm{~nm}$,spectral first derivative trough $942 \mathrm{~nm}$ and peak $758 \mathrm{~nm}$, normalized spectral index maximum NDSI[758,762] and ratio spectral index maximum RSI [758,762] are chosen as modeling parameters at flagging stage. The equation between the predicted and measured values is $\mathrm{y}=0.529 \mathrm{x}+1.8115$, and the value of $\mathrm{R}^{2}$ and RMSE are 0.53 and $0.331 \%$ respectively.

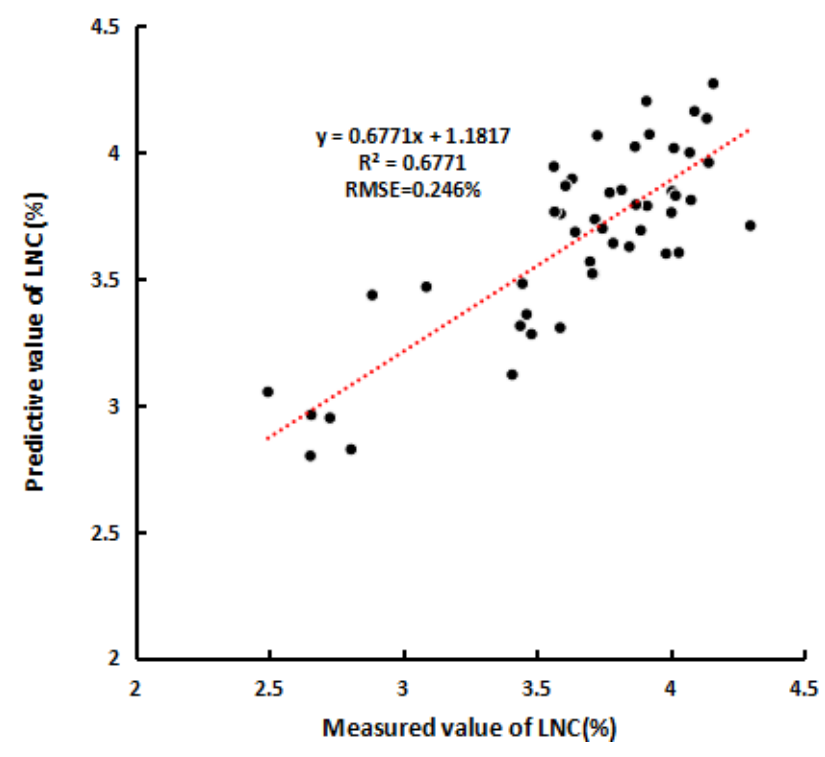

Fig.16 Relationship between predicted LNC and measured LNC at flowering stage

After correlation analysis between spectral and leaf nitrogen content, the original spectral trough $706 \mathrm{~nm}$ and the peak $854 \mathrm{~nm}$,spectral first derivative trough $530 \mathrm{~nm}$ and peak $758 \mathrm{~nm}$, normalized spectral index maximum NDSI[502,574] and ratio spectral index maximum RSI [502,574] are chosen 
as modeling parameters at flowering stage. The equation between the predicted and measured values is $\mathrm{y}=0.6671 \mathrm{x}+1.1817$, and the value of $\mathrm{R}^{2}$ and RMSE are 0.68 and $0.246 \%$ respectively.

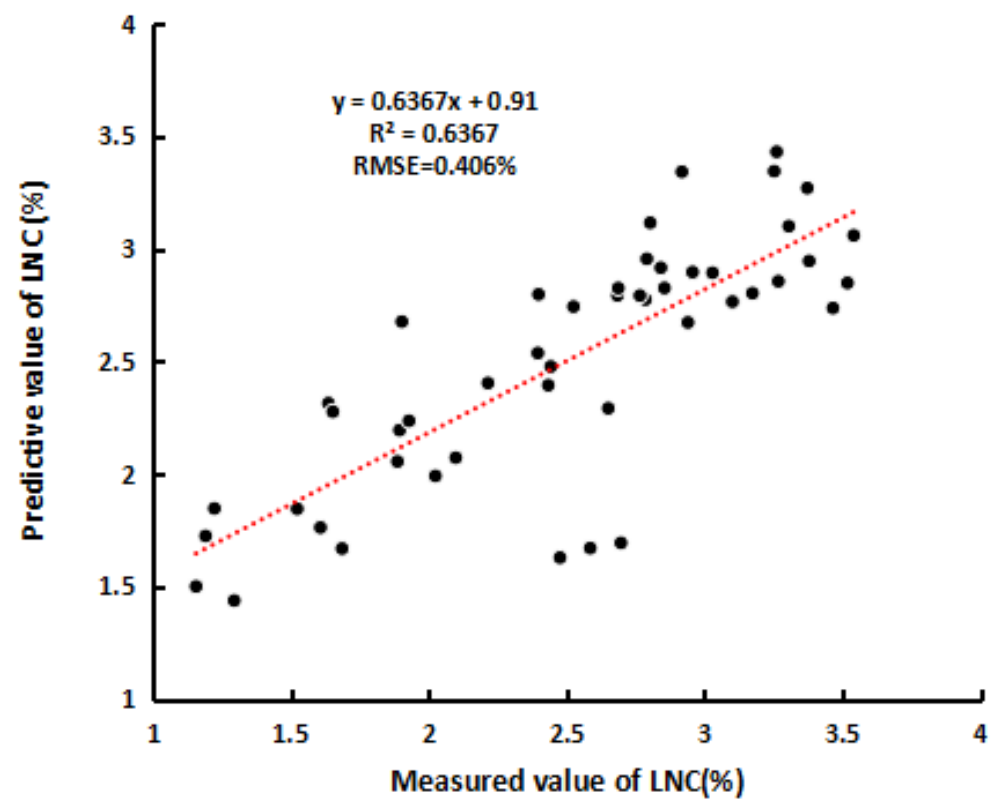

Fig.17 Relationship between predicted LNC and measured LNC at grain filling stage

After correlation analysis between spectral and leaf nitrogen content, the original spectral trough $714 \mathrm{~nm}$ and the peak $786 \mathrm{~nm}$,spectral first derivative trough $682 \mathrm{~nm}$ and peak $738 \mathrm{~nm}$, normalized spectral index maximum NDSI[746,754] and ratio spectral index maximum RSI [746,754] are chosen as modeling parameters at grain filling stage. The equation between the predicted and measured values is $\mathrm{y}=0.6367 \mathrm{x}+0.91$, and the value of $\mathrm{R}^{2}$ and RMSE are 0.64 and $0.406 \%$ respectively.

\subsection{Model validation}

At each stage of growth, we first selected the spectra of the original spectral positive correlation maximum and negative correlation maximum ,the spectra of first derivative positive correlation maximum and negative correlation maximum, a group of spectra with the greatest NDSI correlation value and a group of spectra with the greatest RSI correlation value, with a total of six parameters. And then the Leave a cross-validation method was used to validate the estimation model of nitrogen content in leaves of winter wheat leaves at different growth stages. The coefficient of determination (R2) and the root mean square error (RMSE) were chosen as the indexes to evaluate the validation accuracy. 


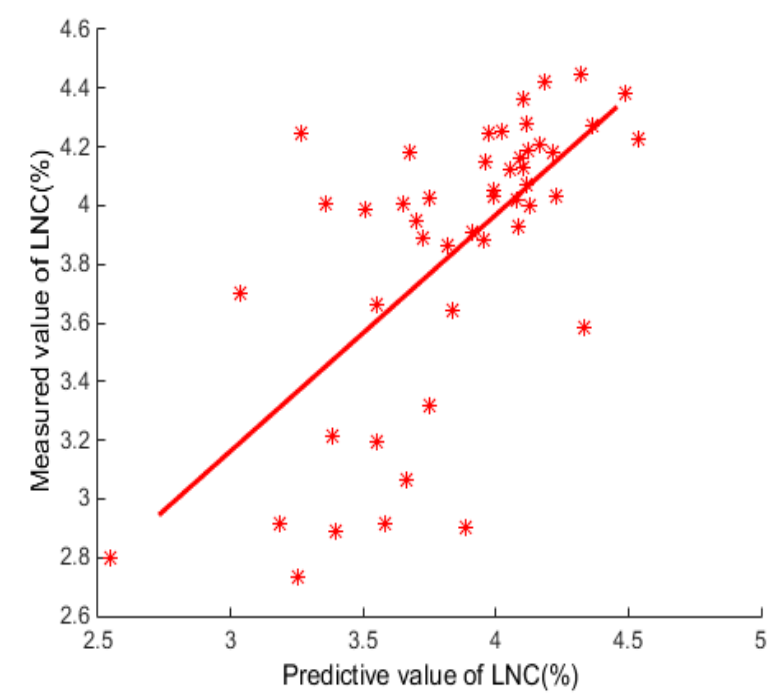

Fig.18 Relationship between predicted LNC and measured LNC at flagging stage

At flagging stage, the original spectral trough $634 \mathrm{~nm}$ and the peak $798 \mathrm{~nm}$,spectral first derivative trough $942 \mathrm{~nm}$ and peak $758 \mathrm{~nm}$, normalized spectral index maximum NDSI[758,762] and ratio spectral index maximum RSI [758,762] are chosen as model validation parameters. The value of R2 and RMSE are 0.4381 and $0.3695 \%$ respectively.

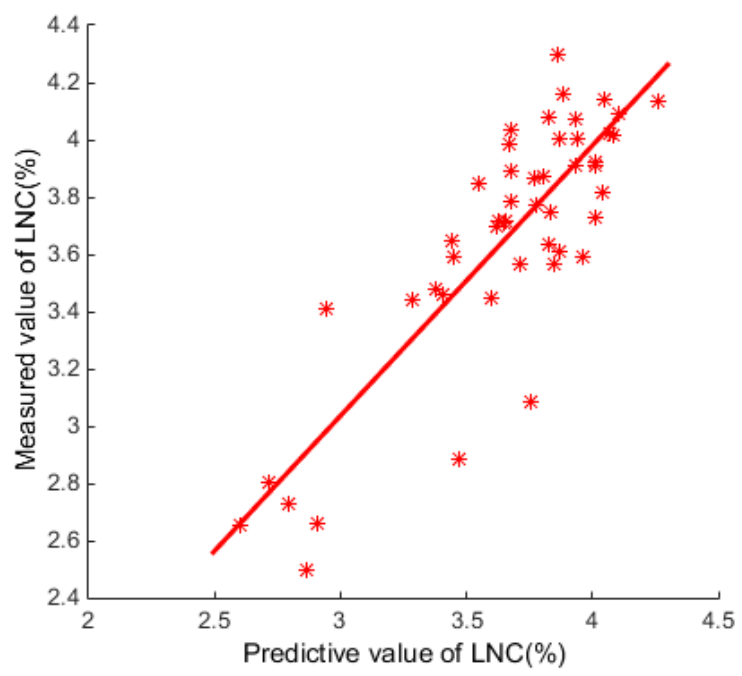

Fig.19 Relationship between predicted LNC and measured LNC at flowering stage

At flowering stage, the original spectral trough $706 \mathrm{~nm}$ and the peak $854 \mathrm{~nm}$,spectral first derivative trough $530 \mathrm{~nm}$ and peak $758 \mathrm{~nm}$, normalized spectral index maximum NDSI[502,574] and ratio spectral index maximum RSI $[502,574]$ are chosen as model validation parameters. The value of R2 and RMSE are 0.7085 and $0.2347 \%$ respectively. 


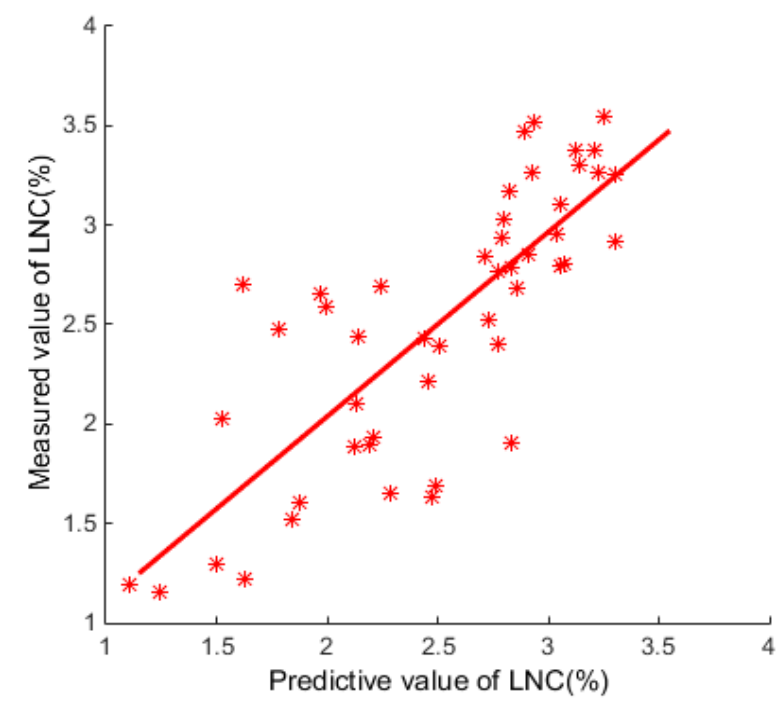

Fig.20 Relationship between predicted LNC and measured LNC at grain filling stage

At grain filling stage, the original spectral trough $714 \mathrm{~nm}$ and the peak $786 \mathrm{~nm}$,spectral first derivative trough $682 \mathrm{~nm}$ and peak $738 \mathrm{~nm}$, normalized spectral index maximum NDSI[746,754] and ratio spectral index maximum RSI [746,754] are chosen as model validation parameters. The value of R2 and RMSE are 0.6352 and $0.40869 \%$ respectively.

\section{Conclusions}

(1) At different growth stages of winter wheat, we first analyze the correlation between the original spectrum, the first derivative of the spectrum, the normalized spectral index (NDSI), the ratio spectral index(RSI) and the leaf nitrogen content, and then screen out the best sensitive bands. these sensitive bands of the flagging stage are as follows: the original spectral $634 \mathrm{~nm}$ and $798 \mathrm{~nm}$,spectral first derivative $942 \mathrm{~nm}$ and $758 \mathrm{~nm}$, NDSI[758,762] and RSI [758,762]. these sensitive bands of the flowering stage are as follows: the original spectral $706 \mathrm{~nm}$ and $854 \mathrm{~nm}$,spectral first derivative $530 \mathrm{~nm}$ and $758 \mathrm{~nm}$, NDSI [502,574] and RSI [502,574]. these sensitive bands of the grain filling stage are as follows: the original spectral $714 \mathrm{~nm}$ and $786 \mathrm{~nm}$, spectral first derivative $682 \mathrm{~nm}$ and $738 \mathrm{~nm}$, NDSI[746,754] and RSI [746,754].

(2) For these three growth stages of flagging stage, flowering stage and grain filling stage, the coefficient of determination $\left(\mathrm{R}^{2}\right.$ ) and the root mean square error (RMSE) of the model building were $0.53,0.68,0.64$ and $0.331 \%, 0.246 \%$ and $0.406 \%$ respectively. The modeling coefficient of determination of the flowering stage is the biggest in these three growth stages, indicating that the flowering stage is the best growth stage for monitoring the nitrogen nutrition of winter wheat leaves by hyperspectral remote sensing.

(3) For these three growth stages of flagging stage, flowering stage and grain filling stage, the coefficient of determination $\left(\mathrm{R}^{2}\right)$ and the root mean square error (RMSE) of model validation were $0.441,0.71,0.64$ and $0.369 \%, 0.235 \%$ and $0.410 \%$ respectively. The results show that the estimation of the nitrogen content of winter wheat plant by using partial least squares method has high accuracy and good reliability. Therefore, it is feasible to monitor the nitrogen content of winter wheat by hyperspectral remote sensing image of UAV. 


\section{Acknowledgments}

This work was supported in part by the National Key Research and Development Programs (2016YFD0300602) ,National Natural Science Foundation of China (No.41601346), Surveying and mapping geographic information public industry scientific research projects(201512010) .

\section{References}

1. Wang Jihua, Zhao Chunjiang, Huang Wenjiang, et al. Quantitative agricultural remote sensing [M]. Beijing: Science Press, 2008.

2. Pinter Jr P J, Hatfield J L, Schepers J S, et al. Remote sensing for crop management[J]. Potogrammetric Engineering \& Remote Sensing, 2003, 69(6): 647-664.

3. Hansen P M, Schjoerring J K. Reflectance measurement of canopy biomass and nitrogen status in wheat crops using normalized difference vegetation indices and partial least squares regression[J]. Remote sensing of environment, 2003, 86(4): 542-553.

4. Feng W, Yao X, Zhu Y, et al. Monitoring leaf nitrogen status with hyperspectral reflectance in wheat[J]. European Journal of Agronomy, 2008, 28(3): 394-404.

5. Clevers J, Kooistra L. Using hyperspectral remote sensing data for retrieving total canopy chlorophyll and nitrogen content[C]//Hyperspectral Image and Signal Processing: Evolution in Remote Sensing (WHISPERS), 20113 rd Workshop on. IEEE, 2011: 1-4.

6. Zhang Xiaoyuan, Zhang Lifu, Zhang Xia, et al. The sensitivity study of retrieving leaf nitrogen content of Winter Wheat with different spectral vegetation index [J]. Chinese agricultural science, 2017, 50 (3): 474-485.

7. Zhu Xinkai, Sheng Haijun, Gu Jing, et al. Preliminary Study on Predicting Chlorophyll and Nitrogen Content in Wheat Leaves Using SPAD Value [J]. Journal of Wheat Crops, 2005, 25 (2): 46-50.

8. Li Yingxue, Zhu Yan, Tian Yongchao, et al.Quantitative relationship between leaf nitrogen content and canopy reflectance spectral index [J]. proceedings of the crop, 2006, 26 (3): 3463-3469.

9. Sun Yanxin, Wang Jihua, Li Baoguo, et al. Establishment and validation of GRNN hyperspectral remote sensing model for retrieving winter wheat leaf nitrogen content based on GA [J]. Bulletin of soils, 2007, 38 (3): 508-512.

10. Zhang Guosheng, Xu Tongyu, Yu FengHua, et al. Rice leaf nitrogen Hyperspectral Estimation and inversion based on the model[J]. Zhejiang Journal of Agricultural Sciences, 2017, 29 (5): 845-849.

11. Wang Jihua, Huang Wenjiang, Lao Cai Lian, et al.Using wheat canopy reflectance spectra to retrieve the vertical distribution of nitrogen by PLS algorithm[J]. Spectroscopy and spectral analysis, 2007, 27 (7): 1319-1322.

12. Ju Changhua. Monitoring nitrogen status and growth characteristics of wheat using ground air hyperspectral remote sensing [D]. Nanjing Agricultural University, 2008.

13. Zhai Qingyun, Zhang Juanjuan, Xiong Shuping, et al. hyperspectral differences and monitoring model construction of nitrogen content in wheat leaves based on different soil texture [J].

14. Li Fenling, Chang Qingrui, Shen Jian, et al. Estimation of nitrogen content in winter wheat leaves with wide band reflectance of simulated multispectral satellite [J].Chinese Journal of agricultural machinery, 2016, 47 (2): 302-308.

15. Wang Renhong, Song Xiaoyu and Li Zhenhai. Estimation of nitrogen nutrition index of Winter Wheat Based on Hyperspectral analysis [J]. Journal of agricultural engineering, 2014, 30 (19): 191-198.

16. Yang Fuqin, Dai Huayang, Feng Haikuan, et al. Estimation of Plant Nitrogen Content in Winter Wheat Using Hyperspectral based on Akaike information criterion [J]. Journal of Agricultural Engineering, 2016, 32 (23): 161167.

17. H Liu, H Zhu,P Wang, et al. Quantitative modeling for leaf nitrogen content of winter wheat using UAV-based hyperspectral data [M]. Taylor \& Francis, Inc. 2017.

18. Li Binbin, Li Zhanbin, Yu Tao, et al. Study on fractal dimension of vegetation cover in watershed based on normalized vegetation index [J]. Journal of Agricultural Engineering, 2014, 30 (15): 239-247. 
19. Tian Y C, Yao X, Yang J, et al. Assessing newly developed and published vegetation indices for estimating rice leaf nitrogen concentration with ground-and space-based hyperspectral reflectance[J]. Field Crops Research, 2011, 120(2): 299-310.(15)

20. Luo Pi, Guo Jichang, Li Qiang, et al. Discussion on Modeling Based on partial least squares regression [J]. Journal of Tianjin University: Natural Science and engineering technology, 2002, 35 (6): 783-786. 